\title{
Clinical effect of therapeutic hemapheresis and plasmapheresis therapy in blood diseases.
}

\author{
Zhen-Hua Bao ${ }^{1,2 \#}$, Jun-Hong Yu${ }^{3 \#}$, Hong-Guo $\mathrm{Zhao}^{4}$ * \\ ${ }^{1}$ Department of Medicine of Qingdao University, Qingdao, Shandong, PR China \\ ${ }^{2}$ Department of Hematology, the People's Hospital of Haiyang, Haiyang, Shandong, PR China \\ ${ }^{3}$ The Affiliated Weihai Second Municipal Hospital of Qingdao University, Weihai, Shandong, PR China \\ ${ }^{4}$ Department of Hematology, Affiliated Hospital of Qingdao University, Qingdao, Shandong, PR China \\ \#These authors contributed equally to this paper
}

\begin{abstract}
Objective: This study aims to discuss the clinical effect of therapeutic hemapheresis+plasmapheresis therapy in patients with blood diseases.

Methods: A total of 140 patients with blood diseases were chosen in our hospital from November 2015 to August 2017 as the research object. Therapeutic hemapheresis+plasmapheresis therapy was adopted, and the final therapeutic effect and laboratory index results were observed.

Results: During RBC leukapheresis, RBC, Hb level, and Hct level of patients decreased significantly compared with those of before the treatment $(P<0.05)$. During leukocyte leukapheresis, PLT and WBC levels of patients declined dramatically compared with those before the treatment $(P<0.05)$. During PLT leukapheresis, the PLT level of patients was considerably lower than that before the treatment $(P<0.05)$. Therapeutic effect evaluation was conducted after therapeutic hemapheresis. The RBC leukapheresis efficiency and PLT leukapheresis efficiency both reached $100.00 \%$. Leukocyte leukapheresis efficiency reached 96.63\%. Thus, outstanding clinical effects were observed. Peripheral blood leucocytes of 6 patients with HLL decreased sharply after the treatment. Patients reported mild nausea and dizziness. Therapeutic hemapheresis was continuously offered after the corresponding treatment, which contributed a sharp reduction of leukocytes. No untoward effects were observed.

Conclusions: Therapeutic hemapheresis+plasmapheresis therapy to patients with blood diseases achieved outstanding therapeutic effects, and can offer full guarantee to symptom relief and safety improvement, thus effectively increasing comprehensive therapeutic effect of patients with blood disease.
\end{abstract}

Keywords: Blood diseases, Therapeutic hemapheresis, Plasmapheresis D.

Accepted on December 26, 2017

\section{Introduction}

Recently, therapeutic hemapheresis and plasmapheresis of patients are widely used in clinical treatment and can achieve outstanding clinical effects $[1,2]$. This study aimed to discuss the clinical value of therapeutic hemapheresis+plasmapheresis to increase the therapeutic effect of patients with blood diseases.

\section{Information and Methods}

\section{General information}

In this study, 140 patients with blood diseases were chosen in our hospital from November 2015 to August 2017 as the research group. There were 82 males and 58 females who aged from $16 \sim 77 \mathrm{y}$ old $(55.69 \pm 2.35 \mathrm{y}$ in average $)$. Among the 140 patients, 136 patients received the primary treatment, while 4 cases belonged to recurrence. Complete examinations were performed to all patients. Furthermore, 120 patients have leukemia, (40 patients with chronic myeloid leukemia, 2 patients with chronic lymphatic leukemia, 28 patients with acute lymphoblastic leukemia, and 50 patients of acute nonlymphocytic leukemia), 10 patients have essential thrombocythemia, 10 patients have polycythemia vera, 10 patients have multiple myeloma, and 22 patients have thrombotic thrombocytopenic purpura.

\section{Methods}

Collection program was set according to diagnosis results and clinical conditions of patients with blood diseases. Before 
sample collection of patients, routine clinical examination was performed including height, blood routine examination, and weight. Subsequently, double-needle continuous circulation was created at median cubital vein or bilateral femoral vein [3]. Therapeutic hemapheresis includes three types, namely, leukocyte leukapheresis, RBC leukapheresis, and PLT leukapheresis. Leukocyte leukapheresis was accomplished by lymphocyte apheresis or granulocyte apheresis, RBC leukapheresis was accomplished by red blood cell separation program, and PLT leukapheresis was accomplished by thrombocyte separation program [4]. In clinics, 90 patients chose leukocyte leukapheresis and a total of 178 aphereses were performed. All patients had high acute leukemia. Before preparing for treatment, peripheral blood WBC level of patients was $123.8 \times 10^{9} / \mathrm{L}$ and $639.16 \times 10^{9} / \mathrm{L}$. During treatment, blood volume of each treatment was in the range of 5520-7000 ml. Besides, 16 patients chose PLT leukapheresis and a total of 46 aphereses were performed. Before preparing for treatment, peripheral blood PLT level of patients ranged between $1050 \times 10^{9} / \mathrm{L}$ and $3553 \times 10^{9} / \mathrm{L}$ [5]. During treatment, blood volume of each treatment was in the range of 4000 5690 $\mathrm{ml}$. Lastly, 12 patients chose median red blood cells, and a total of 14 aphereses were performed. Before preparing for treatment, peripheral blood hemoglobin of patients was tested; it was in the range of 185-202 g/L. During treatment, blood volume of each treatment was in the range of 4000-5690 ml.

During PE operation, time of plasmapheresis was determined according to disease conditions and clinical effect of patients. Each plasmapheresis volume was controlled at 1.0 1.5 times of plasma volume, generally in the range of 1500 3000 ml.
Fresh frozen plasma and normal saline were used as displacement liquid [6]. Moreover, patients were asked to orally take $10 \%$ calcium gluconate or offered continuous intravenous drip of mixing $10 \% 30 \mathrm{ml}$ calcium gluconate into $5 \%$ glucose saline. Twenty-two patients had plasmapheresis, and the procedure was performed for a total of 244 times. Specifically, 6 patients had 40-46 times of plasmapheresis, 10 patients had 14-18 times, 1 patient had 26 times, 1 patient had 8 times, and 1 patient had 25 times. After blood separation treatment, all patients were treated by other routine interventions according to symptoms.

\section{Statistical analysis}

All observation data were treated by SPSS22.0. Measurement data and enumeration data were expressed in $\overline{\mathrm{x}} \pm \mathrm{s}$ and $\%$, and were examined by t-test and $\chi^{2}$-test, respectively. $\mathrm{P}<0.05$ indicated statistically significant difference.

\section{Results}

\section{Indexes after therapeutic hemapheresis}

During RBC leukapheresis, RBC, Hb level, and Hct level of patients decreased significantly compared with those of before the treatment $(\mathrm{P}<0.05)$. During leukocyte leukapheresis, PLT level and WBC level of patients declined dramatically compared with those before the treatment $(\mathrm{P}<0.05)$. In PLT leukapheresis, the PLT level of patients was considerably lower than that before the treatment $(\mathrm{P}<0.05)$. Details are shown in Tables 1 and 2 .

Table 1. Comparison of clinical indexes before therapeutic hemapheresis $(\bar{x} \pm s)$.

\begin{tabular}{|c|c|c|c|c|c|c|}
\hline Groups & Cases/times & $\operatorname{RBC}\left(\times 10^{12} / L\right)$ & $\mathrm{Hb}(\mathrm{g} / \mathrm{L})$ & Hct (\%) & WBC $\left(\times 10^{9} / L\right)$ & PLT (× 109/L) \\
\hline $\mathrm{RBC}$ & $12 / 14$ & $6.19 \pm 0.39$ & $202.49 \pm 22.39$ & $56.82 \pm 3.73$ & $11.69 \pm 2.63$ & $245.22 \pm 41.35$ \\
\hline Leukocyte & $90 / 178$ & $3.16 \pm 0.16$ & $99.02 \pm 8.13$ & $27.29 \pm 2.15$ & $215.29 \pm 37.13$ & $208.79 \pm 40.25$ \\
\hline PLT & $16 / 46$ & $3.69 \pm 0.21$ & $116.96 \pm 14.95$ & $37.15 \pm 0.83$ & $12.59 \pm 2.32$ & $961.13 \pm 248.85$ \\
\hline
\end{tabular}

Table 2. Comparison of clinical indexes after therapeutic hemapheresis $(\bar{x} \pm s)$.

\begin{tabular}{|c|c|c|c|c|c|c|}
\hline Groups & Cases/times & $\operatorname{RBC}\left(\times 10^{12} / L\right)$ & $\mathrm{Hb}(g / L)$ & Hct (\%) & WBC $\left(\times 10^{9} / L\right)$ & PLT (× 109/L) \\
\hline RBC & $12 / 14$ & $4.79 \pm 0.29$ & $143.15 \pm 12.15$ & $44.83 \pm 2.62$ & $10.69 \pm 2.15$ & $248.79 \pm 42.05$ \\
\hline Leukocyte & $90 / 178$ & $2.76 \pm 0.19$ & $89.33 \pm 5.21$ & $25.29 \pm 1.63$ & $45.29 \pm 7.15$ & $158.79 \pm 39.02$ \\
\hline PLT & $16 / 46$ & $3.61 \pm 0.30$ & $107.69 \pm 11.15$ & $37.15 \pm 1.82$ & $11.52 \pm 2.43$ & $261.13 \pm 68.72$ \\
\hline
\end{tabular}

\section{Clinical effect after therapeutic hemapheresis}

By observing and analyzing therapeutic effect evaluation after therapeutic hemapheresis, the RBC leukapheresis efficiency and PLT leukapheresis efficiency both reached $100.00 \%$, whereas the leukocyte leukapheresis efficiency reached $96.63 \%$, thereby showing outstanding clinical effects. Results are shown in Table 3. Peripheral blood leucocytes of 6 patients with HLL decreased sharply after the treatment. Patients reported mild nausea and dizziness. Therapeutic hemapheresis was continuously offered after the corresponding treatment, which contributed to a sharp reduction of leukocytes. No untoward effects were observed. 
Table 3. Clinical statistics on total therapeutic effects.

\begin{tabular}{llllc}
\hline Groups & Cases/times & Significantly effective & Effective & Ineffective \\
\hline RBC leukapheresis & $12 / 14$ & 14 & 0 & 0 \\
\hline Leukocyte leukapheresis & $90 / 178$ & 140 & 32 & 6 \\
\hline PLT leukapheresis & $16 / 46$ & 44 & 2 & 00.00 \\
\hline
\end{tabular}

\section{Statistics on PE therapeutic effects}

Clinical effects after PE treatment of patients with myeloma are shown in Table 4.

Table 4. Statistics on PE therapeutic effect of patients with blood diseases.

\begin{tabular}{lll}
\hline Indexes & Before & After \\
\hline ESR $(\mathrm{mm} / \mathrm{h})$ & $88.83 \pm 17.36$ & $49.72 \pm 22.29$ \\
\hline $\mathrm{GLB}(\mathrm{g} / \mathrm{L})$ & $83.63 \pm 15.49$ & $43.69 \pm 15.39$ \\
\hline
\end{tabular}

\section{Discussion}

Therapeutic hemapheresis+plasmapheresis therapy achieved outstanding effect on patients with blood diseases [7]. Based on analysis of treatment principle, therapeutic hemapheresis +plasmapheresis eliminated the pathological components in blood and effectively replaced them by using a blood separator. It supplemented blood volume by replacing liquid and finally relieving disease symptoms significantly [8,9]. This treatment was mainly monitored by computer and the in vitro circulation volume could be reduced dramatically. Moreover, the treatment was safe and had outstanding therapeutic effect.

In this study, RBC, Hb level, and Hct level of patients after RBC leukapheresis decreased significantly compared with those of before the treatment $(\mathrm{P}<0.05)$. During leukocyte leukapheresis, PLT and WBC levels of patients declined dramatically compared with those before the treatment $(\mathrm{P}<0.05)$. During PLT leukapheresis, PLT level of patients was considerably lower than before the treatment $(\mathrm{P}<0.05)$. By observing and analyzing therapeutic effect evaluation after therapeutic hemapheresis, the RBC leukapheresis efficiency and PLT leukapheresis efficiency both reached $100.00 \%$, whereas the leukocyte leukapheresis efficiency reached $96.63 \%$, thereby showing outstanding clinical effects. Peripheral blood leucocytes of 6 patients with HLL decreased sharply after the treatment. Patients reported mild nausea and dizziness. Therapeutic hemapheresis was continuously offered after the corresponding treatment, which contributed to a sharp reduction of leukocytes [10]. No untoward effects were observed. These results proved the clinical value of therapeutic hemapheresis+ plasmapheresis therapy.

\section{Conclusion}

The therapeutic hemapheresis + plasmapheresis therapy on patients with blood diseases showed outstanding and quick therapeutic effect and effectively increased the comprehensive therapeutic effect on patients with blood disease.

\section{References}

1. Tarantal AF, Skarlatos SI. Center for fetal monkey gene transfer for heart, lung, and blood diseases: an NHLBI resource for the gene therapy community. Hum Gene Ther 2012; 23: 1130-1135.

2. Latchoumi TP, Parthiban L. Abnormality detection using weighed particle swarm optimization and smooth support vector machine. Biomed Res India 2017; 28: 4749-4751.

3. Li QY, Yu F, Zhou FD, Zhao MH. Plasmapheresis is associated with better renal outcomes in lupus nephritis patients with thrombotic microangiopathy. Medicine (Baltimore) 2016; 95: 3595.

4. Nguyen TC, Kiss JE, Goldman JR, Carcillo JA. The role of plasmapheresis in critical illness. Crit Care Clin 2012; 28: 453-468.

5. Noreen S, Shahzad S, Qadir MA. Synthesis of 5fluorouracil derivatives for enhanced blood circulation. Lat Am J Pharm 2017; 36: 2267-2272.

6. Cortese I, Chaudhry V, So YT, Cantor F, Cornblath DR, Rae-Grant A. Evidence-based guideline update: Plasmapheresis in neurologic disorders. Neurology 2011; 76: 294-300.

7. Chen LZ, Li XH. Evaluation of the inhibition of ear-nosethroat (ENT) diseases treatment drug mecobalamin on drug-metabolizing enzymes (DMEs). Lat Am J Pharm 2017; 36: 2302-2305.

8. Chang B, Tholpady A, Huang RSP, Nedelcu E, Bai Y. Clinical and serological responses following plasmapheresis in bullous pemphigoid: two case reports and a review of the literature. Blood Transfus 2014; 12: 269-275.

9. Polat Y. Effects of CoQ10 and zinc supplemented trainings upon some blood parameters. Biomed Res India 2017; 28: 4287-4293.

10. Rosa-Bray M, Wisdom C, Wada S, Johnson BR, GrifolsRoura V, Grifols-Lucas V. Prospective multicentre study of the effect of voluntary plasmapheresis on plasma cholesterol levels in donors. Vox Sang 2013; 105: 108-115.

\section{*Correspondence to}

Hong-Guo Zhao

Department of Hematology

Affiliated Hospital of Qingdao University

PR China 